\title{
Differences in stability of seed-associated microbial assemblages in response to invasion by phytopathogenic microorganisms
}

Samir Rezki, Claire Campion, Beatrice lacomi-Vasilescu, Anne Preveaux, Youness Toualbia, Sophie Bonneau, Martial Briand, Emmanuelle Laurent, Gilles Hunault, Philippe Simoneau, Marie-Agnès Jacques, Matthieu Barret

Seeds are involved in the vertical transmission of microorganisms from one plant generation to another and consequently act as reservoirs for the plant microbiota. However, little is known about the structure of seed-associated microbial assemblages and the regulators of assemblage structure. In this work, we have assessed the response of seed-associated microbial assemblages of Raphanus sativus to invading phytopathogenic agents, the bacterial strain Xanthomonas campestris pv. campestris (Xcc) 8004 and the fungal strain Alternaria brassicicola Abra43. According to the indicators of bacterial (16S rRNA gene and gyrB sequences) and fungal (ITS1) diversity employed in this study, seed transmission of the bacterial strain Xcc 8004 did not changed the overall composition of resident microbial assemblages. In contrast seed transmission of Abra43 strongly modified the richness and structure of fungal assemblages without affecting bacterial assemblages. The sensitivity of seed-associated fungal assemblage to Abra43 is mostly related to changes in relative abundance of closely related fungal species that belong to the Alternaria genus. Variation in stability of the seed microbiota in response to Xcc and Abra43 invasions could be explained by differences in seed transmission pathways employed by these micro-organisms, which ultimately results in divergence in spatiotemporal colonization of the seed habitat. 
1 Title : Differences in stability of seed-associated microbial assemblages in response to

2 invasion by phytopathogenic microorganisms

3 Samir Rezki ${ }^{1}$, Claire Campion ${ }^{2}$, Beatrice Iacomi-Vasilescu $^{3}$, Anne Préveaux ${ }^{1}$, Youness Toualbia ${ }^{2}$,

4 Sophie Bonneau ${ }^{1}$, Martial Briand ${ }^{1}$, Emmanuelle Laurent $^{5}$, Gilles Hunault ${ }^{4}$, Philippe Simoneau ${ }^{2}$,

5 Marie-Agnès Jacques ${ }^{1}$ and Matthieu Barret ${ }^{1 *}$

7 Authors affiliation:

$8{ }^{1}$ INRA, UMR1345 Institut de Recherches en Horticulture et Semences, SFR4207 QUASAV, F-

949071 Beaucouzé, France

10 2Université d'Angers, UMR1345 Institut de Recherches en Horticulture et Semences, Beaucouzé,

11 France

12 3USAMV, 59 Bd Marasti, Ro-71331 Bucharest, Romania

13 `Université d'Angers, Laboratoire d'Hémodynamique, Interaction Fibrose et Invasivité tumorale

14 Hépatique, UPRES 3859, IFR 132, France

$15{ }^{5}$ FNAMS, Brain-sur-1'Authion, France

16

*Corresponding author:

Matthieu Barret

19

42 rue Georges Morel, Beaucouzé, 49071, France

matthieu.barret@angers.inra.fr 


\section{Abstract (199 words)}

26 Seeds are involved in the vertical transmission of microorganisms from one plant generation to

27 another and consequently act as reservoirs for the plant microbiota. However, little is known about

28 the structure of seed-associated microbial assemblages and the regulators of assemblage structure.

In this work, we have assessed the response of seed-associated microbial assemblages of Raphanus sativus to invading phytopathogenic agents, the bacterial strain Xanthomonas campestris pv. campestris (Xcc) 8004 and the fungal strain Alternaria brassicicola Abra43. According to the indicators of bacterial (16S rRNA gene and $g y r B$ sequences) and fungal (ITS1) diversity employed in this study, seed transmission of the bacterial strain Xcc 8004 did not changed the overall composition of resident microbial assemblages. In contrast seed transmission of Abra43 strongly modified the richness and structure of fungal assemblages without affecting bacterial assemblages. The sensitivity of seed-associated fungal assemblage to Abra43 is mostly related to changes in relative abundance of closely related fungal species that belong to the Alternaria genus. Variation in stability of the seed microbiota in response to Xcc and Abra43 invasions could be explained by differences in seed transmission pathways employed by these micro-organisms, which ultimately results in divergence in spatio-temporal colonization of the seed habitat. 


\section{Introduction (893 words)}

Seeds are not only carriers of plants genetic information but are also involved in the vertical transmission of microorganisms from one plant generation to another and consequently act as reservoirs for the plant microbiota (Baker and Smith, 1966; Nelson, 2004). The activity of seedassociated microbial assemblages is significant for plant growth and plant health since these microbial assemblages may release seed dormancy through production of cytokinins (Goggin et al., 2015) or limit the installation of microbial invader (Bacilio-Jimenez et al., 2001). Although transmission of microorganisms from plant to seed is the primary source of inoculum for the plant, relatively little is known about the structure of seed-associated microbial assemblages and the regulators of assemblage structure (Johnston-Monje and Raizada, 2011; van Overbeek et al., 2011; Lopez-Velasco et al., 2013; Links et al., 2014; Barret et al., 2015; Klaedtke et al., 2015). Seeds acquire their microbiome by three majors' pathways: (i) internal transmission through the vascular system, (ii) floral transmission by the stigma and (iii) external transmission via contact of the seed with microorganisms present on fruits, flowers or residues (Maude, 1996). According to the transmission pathway, seed-borne microorganisms can therefore be located on different micro-habitats ranging from the testa to the embryo (Singh and Mathure, 2004; Dutta et al., 2012; Tancos et al., 2013). While the internal transmission by the host xylem is restricted to vascular pathogens or endophytic micro-organisms (Maude, 1996), many plant associated microorganisms are potentially transmitted to the seed by the floral pathway (Shade et al., 2013; Aleklett et al., 2014). Indeed, the floral pathway allows the transmission of biocontrol microorganisms (Spinelli et al., 2005) and phytopathogens (Darsonval et al., 2008; Darrasse et al., 2010; Terrasson et al., 2015). Finally, the external pathway is probably the most permissive way of microorganism 
64 transmission from plant to seed, although very little data are currently available in the literature

65 (Ngugi and Scherm, 2006).

66

67

68

69

Owing to the importance of seed transmission in emergence of diseases in new planting areas, the processes involved in the transmission of microorganisms from plant to seed have been mainly documented for phytopathogenic agents. The molecular determinants involved in successful transmission of microorganisms from plant to seed have been notably studied in bacteria related to the Xanthomonas genus (Darsonval et al., 2008; Darsonval et al., 2009; Darrasse et al., 2010; Dutta et al., 2014) and fungi that belonged to Alternaria brassicicola (Pochon et al., 2012; Pochon et al., 2013). Key molecular determinants like the bacterial Type III Secretion System (Darsonval et al., 2008), bacterial adhesins (Darsonval et al., 2009), fungal class III histidinekinase or dehydrin like proteins (Pochon et al., 2013) have been shown to be involved in seed transmission. In turn, seeds may respond to pathogen transmission through activation of plant defenses and subsequent repression of seed maturation pathways (Terrasson et al., 2015).

Although the host immune system is a decisive environmental filter to limit the installation of an invader (Jones and Dangl, 2006), the host-associated microbial community may also strongly prevent this invasion (Mendes et al., 2011). The resistance of microbial community to invasion is linked to its level of diversity, since highly diverse microbial community are usually less sensitive to invasion (Jousset et al., 2011; van Elsas et al., 2012) as a result of enhanced competition for resources within species-rich community (Mallon et al., 2015a; Mallon et al., 2015b; Wei et al., 2015). Because of its relative low microbial diversity (Lopez-Velasco et al., 2013; Links et al., 2014; Barret et al., 2015; Klaedtke et al., 2015) compared to other plant habitat such as the phyllosphere (Vorholt, 2012) or the rhizosphere (Hacquard et al.), mature seed is an interesting experimental model to study biological disturbance. Moreover, mature seeds have low moisture 
87 content and are almost metabolically inactive (Dekkers et al., 2015), which suggest that associated

88 micro-organisms are probably dormant and that the structure of microbial assemblages is in a

89 stable state. Hence shift in assemblage structure are likely to reflect the outcome of the response

90 of the microbial assemblage during seed transmission of phytopathogenic micro-organisms.

91 The aim of the present work was to analyze the impact of two microbial invaders, namely

92 the bacterial strain Xanthomonas campestris pv. campestris (Xcc) 8004 and the fungal strain $A$.

93 brassicicola $(A b)$ Abra43 on the genetic structure of microbial assemblages associated to seeds of

94 Raphanus sativus. We choose these two microbial invaders since they differ in their seed

95 transmission pathways. Indeed $X c c$ is mostly transmitted from plant to seeds by the systemic and

96 floral pathways (Cook et al., 1952; Van Der Wolf and Van Der Zouwen, 2010; van der Wolf et

97 al., 2013), while $A b$ is transmitted by the external pathway and thus mostly restricted to the testa

98 (Knox-Davies, 1979). Radish (Raphanus sativus) was used as an experimental system since this

99 plant has a high seed multiplication ratio (1: 500 on averages) and a low microbial diversity

100 compared to other Brassicaceae species (Barret et al., 2015). Profiling of seed-associated microbial

101 assemblages was performed on mature seeds harvested from uninoculated plants or plants

102 inoculated either with Abra43 or Xcc 8004 through sequencing of two bacterial taxonomic markers

103 (16S rRNA gene and $g y r B)$ and one fungal taxonomic marker (ITS1 region of the fungal internal

104 transcribed spacer). This work revealed that the plant pathogenic fungal strain Abra43 had a

105 significant impact on the fungal assemblages, while Xcc 8004 transmission did not impact the 106 structure of seed microbiota. 
107

108

109

110

111

112

113

114

115

116

117

118

119

120

121

122

123

124

125

126

127

128

129

\section{Materials \& Methods (1,861 words)}

\section{Site description, inoculation process and seed collection}

Experiments were performed at the FNAMS experimental station $\left(47^{\circ} 28^{\prime} 12.42^{\prime \prime} \mathrm{N}, 0^{\circ} 23^{\prime} 44.30^{\prime \prime} \mathrm{W}\right.$, Brain-sur-l'Authion, France) in 2013 and 2014 on 3 distinct plots (5 x 10 meters). Each plot was initially sown with Raphanus sativus var. Flamboyant5 on March $28^{\text {th }} 2013$ with a commercial seed lot. According to microbiological and community profiling analyses this seed sample was neither contaminated with Xanthomonas campestris pv. campestris (Xcc) nor Alternaria brassicicola (Barret et al., 2015). One plot (X2013) was spray-inoculated (approximately 100 $\mathrm{ml} . \mathrm{m}^{-2}$ ) at floral stage (June, $18^{\text {th }} 2013$ ) with Xcc strain 8004. Xcc 8004 is a spontaneous rifampicinresistance strain derived from Xcc NCPPB 1145 (Qian et al., 2005). The bacterial strain Xcc 8004 was cultivated on Tryptic Soy Agar (TSA) 100\% (17 g. $1^{-1}$ tryptone, 3 g..$^{-1}$ soybean peptone, 2.5 g..$^{-1}$ glucose, 5 g. $.^{-1} \mathrm{NaCl}, 5$ g..$^{-1} \mathrm{~K} 2 \mathrm{HPO} 4$, and 15 g. $\mathrm{l}^{-1}$ agar) medium with rifampicin $50 \mathrm{mg} . \mathrm{l}^{-1}$ for 2 days at $28^{\circ} \mathrm{C}$. Bacterial colonies of Xcc 8004 were suspended in sterile deionized water at a final concentration of $1.10^{7} \mathrm{CFU} / \mathrm{ml}$. Another plot (A2013) was inoculated with Alternaria brassicicola strain Abra43 (Avenot et al., 2005) at the end of flowering (July 05 ${ }^{\text {th }} 2013$ ) and at silique-formation stage (July $17^{\text {th }} 2013$ ) following protocol described earlier (Iacomi-Vasilescu et al 2008). The last plot (C2013) was not inoculated and subsequently used as a control plot. Plots were watered $4 \mathrm{~h}$ before inoculation in order to have a high relative humidity (approximately $80 \%$ ).

A second experimentation was performed in 2014. Seeds harvested from C2013 were sown on April 2 $2^{\text {th }}, 2014$ following the same experimental design as in 2013. While Alternaria brassicicola was not detected within C2013 sample, a residual contamination of Xcc was observed

(Fig. 1A and Fig. 1C, see results section for further details). The only difference between 2013 
130 and 2014 experiments rely in Xcc 8004 inoculations. While in 2013 plants were inoculated once,

131 two inoculations were performed in 2014 in order to increase the efficiency of Xcc 8004

132 transmission from plant to seed. Inoculation of Xcc 8004 were done at the beginning (June, $18^{\text {th }}$

$1332014)$ and at the end (June, $25^{\text {th }} 2014$ ) of flowering stage. Inoculation with $A b$ (June, $26^{\text {th }} 2014$ and

134 July $7^{\text {th }}$ 2014) was performed in one plot (A2014) using the same protocol as during the 2013

135 experimentation. The last plot (C2014) was left uninoculated. We did not observed any leaf spot

136 symptoms on plants inoculated with Xcc 8004. In contrast, some dark spots on pods were observed

137 on plants inoculated with Alternaria brassicicola Abra43.

138 At the mature seeds stage, seeds from plots C2013, A2013 and X2013 were harvested on

139 September, $^{\text {th }}$ 2013, while seeds from plots C2014, A2014 and X2014 were collected on August,

$14022^{\text {th }}$ 2014. Eighteen plants of each plot were collected and seeds were harvested manually for each

141 individual plant. The remaining plants of each plot were harvested with a threshing machine

142 leading to 6 seed lots (C2013, C2014, A2013, A2014, X2013 and X2014). Each of these seed lots

143 was further divided into subsamples of 1,000 seeds (as assessed by 1,000 seeds weight). Seeds

144 were conserved between one to two months at $9{ }^{\circ} \mathrm{C}$ and $50 \%$ relative humidity prior to DNA

145 extraction.

146

147 Culture-based detection of phytopathogenic agents on seed samples

148 The transmission of Xcc 8004 from plants to seeds was initially evaluated through microbiological

149 analysis of seed lots harvested with the threshing machine. Individual seeds were deposited in a

15096 well-plate and soaked in $200 \mu \mathrm{L}$ of phosphate buffer saline (PBS) supplemented with $0.05 \%$

$151(\mathrm{v} / \mathrm{v})$ of Tween ${ }^{\circledR} 20$ during 2 hours and 30 minutes at room temperature under constant agitation

152 (140 rpm). Then $10 \mu 1$ of each well was spread on TSA $10 \%$ medium supplemented with rifampicin 
$153(50 \mathrm{mg} / \mathrm{l})$. Plates were incubated at $28^{\circ} \mathrm{C}$ for 2 days and the presence of $X c c 8004$ was evaluated

154 for each individual seed. Contamination rate of each seed lot is a mean of six biological replicates,

155 each containing 96 seeds. The transmission of Alternaria brassicicola strain Abra43 (Ab) from

156 plant to seeds was assessed by standard plating technique (Iacomi-Vasilescu et al., 2008). Briefly,

15710 individual seeds were deposited on potato-dextrose agar ( 4 g. $1^{-1}$ potato extract, 20 g. $1^{-1}$ dextrose,

15815 g. $1^{-1}$ agar) and plates were incubated at $25^{\circ} \mathrm{C}$ for 5 days. Alternaria brassicicola isolates were

159 further purified by monospore isolation. Contamination rate of each seed lot is a mean of 6

160 biological replicates, each biological replicate containing 100 seeds (10 plates per seed lot).

161

\section{DNA extraction}

163 DNA extraction were performed according to standard procedures recommended by the 164 International Seed Testing Association (ISTA, https:/www.seedtest.org/en/home.html). Briefly, 165 seeds harvested manually on each plant or 1,000 seeds subsamples harvested mechanically were 166 transferred in sterile tubes containing $25 \mathrm{~mL}$ of PBS supplemented with $0.05 \%(v / v)$ of Tween ${ }^{\circledR}$ 167 20. Samples were incubated during 2 hours and 30 minutes at room temperature under constant 168 agitation $(140 \mathrm{rpm})$. Seed soaking procedures are routinely used to assess the presence of seed169 borne pathogens within seed tissues (Gitaitis \& Walcott, 2007). Indeed, microorganisms located 170 within the seed coat as well as in the funiculus are released in the suspension with such 171 experimental procedure. Suspensions were centrifuged $\left(6000 \mathrm{x} g, 10 \mathrm{~min}, 4^{\circ} \mathrm{C}\right)$ and pellets were 172 resuspended in approximately $2 \mathrm{ml}$ of supernatant and transferred in microtubes. Total DNA was 173 extracted with the Power Soil DNA Kit (MoBio Laboratories) from 148 seed suspensions 174 following procedure described earlier (Barret et al., 2015). 


\section{Molecular detection of phytopathogenic agents on seed samples}

177 Seed transmission of phytopathogenic agents was also monitored by quantitative PCR. The number of copy of the predicted gene $\mathrm{XC}_{-} 1533$ encoding a hypothetical protein was used as an

179 estimator of the number of Xcc 8004 cells per seed samples (Rijlaarsdam et al., 2004). A portion 180 of this gene was amplified with the primer set Zup4F/Zup4R (Table S6). Data normalization 181 between seed samples was performed with a portion of the $16 \mathrm{~S}$ rRNA gene, using the primers 926F/1062R (Table S1). All reactions were performed in $25 \mu 1$ qPCR reaction using $12.5 \mu 1$ of SYBR Green Master Mix (MESA BLUE qPCR MasterMix Plus for SYBR Assay; Eurogentec, Cologne, Germany), $2 \mu \mathrm{l}$ of DNA (10 ng. $\left.\mu \mathrm{l}^{-1}\right)$ and $0.5 \mu \mathrm{L}$ of each primer $(10 \mu \mathrm{M})$. Amplification conditions were $5 \mathrm{~min}$ at $95^{\circ} \mathrm{C}$, followed by 40 two-step cycles of $95^{\circ} \mathrm{C}(15 \mathrm{~s})$ and $60^{\circ} \mathrm{C}(60 \mathrm{~s})$. an estimator of the number of Abra43 cells (Pochon et al., 2013) using the primer set AbraDHN1Tq-F/AbraDHN1-Tq-R and a TaqMan MGB probe (Table S1). Reactions were conducted using StepOnePlus ${ }^{\mathrm{TM}} \mathrm{qPCR}$ system (Applied Biosystems). qPCR reaction were performed in $20 \mu \mathrm{l}$ using $10 \mu \mathrm{l}$ of Master Mix, $2 \mu \mathrm{l}$ of TaqMan probe $(250 \mathrm{nM}), 2 \mu \mathrm{l}$ of each primer $(0.3 \mu \mathrm{M}$ and $0.9 \mu \mathrm{M}$

191 for the forward and the reverse primer respectively) and $2 \mu 1$ of DNA (10 ng. $\left.\mu 1^{-1}\right)$. Amplification conditions were $10 \mathrm{~min}$ at $95^{\circ} \mathrm{C}$, followed by 40 two-step cycles of $95^{\circ} \mathrm{C}(15 \mathrm{~s})$ and $60^{\circ} \mathrm{C}(60 \mathrm{~s})$. the primers ACT 512-F/ACT 783-R (Table S6) and amplification conditions described previously 195 (Carbone and Kohn, 1999).

196

\section{Assessment of microbial diversity}


198 PCR amplification was conducted on 148 DNA samples and 2 artificial microbial community

199 samples containing a mixture of 15 DNA extracted from different bacterial strains (Barret et al 200 2015). The 148 environmental DNA samples correponded to seed samples harvested from 201 individual plants (18 plants per plot) or with a threshing machine for each plot (6 subsamples on 202 average for each plot). Amplification were performed with the primer sets 515F/806R, 203 gyrB_aF64/gyrB_aR353 and ITS1F/ITS2 (Table S1) following procedures described earlier 204 (Barret et al., 2015). Amplicons libraries were mixed with $7.5 \%$ PhiX control according to 205 Illumina's protocols. A total of four sequencing runs were performed for this study with MiSeq 206 Reagent Kits v2 (500 cycles).

Sequence analyses were performed with Mothur v1.31.2 (Schloss et al., 2009) using 208 standard operating procedure (Kozich et al., 2013) described earlier (Barret et al., 2015). Briefly, 16S rRNA gene and gyrB sequences were aligned against the 16S rRNA gene SILVA alignment and a $g y r B$ reference alignment, respectively. Chimeric sequences were detected with UCHIME

211 (Edgar et al., 2011) and subsequently removed from the dataset. Moreover, gyrB sequences 212 containing stop codon were discarded. Taxonomic affiliation of 16S rRNA gene and gyrB 213 sequences was performed with a Bayesian classifier (Wang et al., 2007) (80\% bootstrap confidence 214 score) against the 16S rRNA gene training set (v9) of the Ribosomal Database Project (Cole et al., 215 2009) or against an in-house $g y r B$ database created with sequences retrieved from the IMG 216 database (Markowitz et al., 2012; Barret et al., 2015). Unclassified sequences (0.001\% of the 16S 217 rRNA gene sequences) or sequences belonging to Archaea (0.002\%), chloroplasts $(0.9 \%)$ or 218 mitochondria $(0.004 \%)$ were discarded. Sequences were divided into groups according to their 219 taxonomic rank (level of order) and then assigned to operational taxonomic units (OTUs) at 97\% 220 identity cutoff for 16S rRNA gene and 98\% identity for gyrB sequences. The variable ITS 1 regions 
221 of ITS sequences were extracted with the Perl-based software ITSx (Bengtsson-Palme et al., 2013).

222 Then sequences were clustered at a 97\% identity cut-off using Uclust (Edgar, 2010) and taxonomic

223 affiliation was performed with a Bayesian classifier (Wang et al., 2007) (80\% bootstrap confidence

224 score) against the UNITE database (Abarenkov et al., 2010). To improve the resolution of the

225 taxonomic classification of ITS1 sequences, we performed a reciprocal blast analysis at a

226 minimum cut-off of $97 \%$ with representatives OTUs sequences and available ITS1 sequences of

227 Alternaria type strains (Woudenberg et al., 2013). In order to enhance the reproducibility of

228 community profiles, abundant OTUs (aOTU) representing at least $0.1 \%$ of the library size were

229 used for microbial community analyses (Barret et al., 2015).

230 In order to avoid biases introduced by unequal sampling, total counts were divided by

231 library size of each sample sequenced (McMurdie and Holmes, 2014). Both alpha and beta

232 diversity indexes were calculated with Mothur (Schloss et al., 2009). Richness was defined as the

233 number of different OTUs and aOTUs per sample. Hierarchical clustering of different seed

234 samples was performed using an average linkage method based on Bray-Curtis dissimilarity index

235 (Bray and Curtis, 1957) and on unweighted UniFrac distances (Lozupone and Knight, 2005).

236 Analysis of similarity (ANOSIM) was used to assess the effects of the different conditions on the

237 microbial community structure. Moreover, canonical analysis of principal coordinates (CAP) was

238 conducted to measure the relative influence of $(i)$ the phytopathogenic agent, ( $i i)$ the harvesting

239 year and (iii) the harvesting method on microbial $\beta$-diversity. CAP analyses were performed with

240 the function capscale of vegan.

241 Correlation between aOTUs were calculated with Sparse Correlation for Compositional

242 data algorithm (Friedman and Alm, 2012) implemented in Mothur. Statistical significance of the

243 inferred correlations was assessed with a bootstrap procedure (100 replications). Only correlations 
244 with pseudo $p$-value $\leq 0.001$ were represented in the network using the $\mathrm{R}$ package qgraph

245 (Epskamp et al., 2012). Changes in relative abundance of aOTUs between the different

246 experimental conditions (C, X and A) were assessed with LEfSE (Segata et al., 2011). aOTUs

247 were defined as significantly enriched or depleted in one treatment at a $P$ value $\leq 0.05$ and a LDA

248 score $>2$.

249 All sequences have been deposited in the ENA database under the accession number

250 PRJEB9588.

251 


\section{Results (1,944 words)}

253 The impact of pathogen transmission on the structure of seed-associated microbial assemblages was assessed on radish seed lots harvested in 2013 and 2014 from plots inoculated with the

255 bacterial strain Xanthomonas campestris pv. campestris 8004 (X2013 and X2014 plots), with the 256 fungal strain Alternaria brassicicola Abra43 (A2013 and A2014 plot) and from uninoculated 257 plants (C2013 and C2014 for control plots).

258

\section{Efficient transmission of phytopathogenic microorganisms to seeds}

260

261

262

263

264

265

266

267

268

269

270

271

272

273

274

The seed transmission of $X c c 8004$ and $A b$ Abra43 was first evaluated by standard microbiological approaches on seed samples collected in 2013 and 2014. According to these microbiological analyses, $A b$ was not detected on seeds harvested from control plots (C2013 and C2014), while a residual Xcc contamination of $0.69 \%$ and $0.13 \%$ was observed in seeds from C2013 and C2014 samples, respectively (Fig. 1A and Fig. 1B). However, the incidence of $X c c$ increased significantly in X2013 and X2014 samples ( $P<0.01$, as assessed by 2-sample test for equality of proportions) with $6 \%$ and $26 \%$ of seeds contaminated, respectively (Fig. 1A). $A b$ was not detected in control samples, nevertheless a significant increase of $A b$ incidence $(P<0.01)$ was observed in A2013 and A2014 samples with 54\% and 36\% of seed contaminated (Fig. 1B), respectively.

To confirm these results, qPCR experiments were performed on DNA extracted from seed samples with primers and probes targeting XC_1533, a single-copy gene of Xcc encoding a hypothetical protein (Rijlaarsdam et al., 2004), and $A b D h n 1$, a single-copy gene of $A b$ encoding a dehydrin-like protein (Pochon et al., 2013). A significant increase $(P<0.01$, as assessed by ANOVA with post hoc Tukey's HSD test) in copy number of XC_1533 was observed on X2013

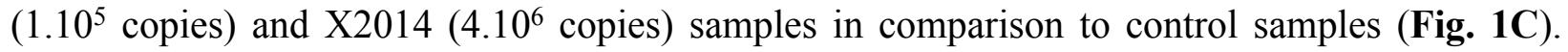


275 Similarly, the number of copy of $A b D h n 1$ also increased $(P<0.01)$ in A2013 $\left(1.5 .10^{10}\right)$ and A2014

$276\left(4.5 .10^{10}\right)$ (Fig. 1D). These differences were not due to variation in DNA amounts between seed

277 samples since the copy numbers of $16 \mathrm{~S}$ rRNA and $\beta$-actin genes were not significantly different

278 between seed samples (Fig. S1). Altogether microbiological and qPCR analyses highlighted an 279 effective transmission of Xcc 8004 and $A b$ Abra43 from plant to seed.

280

281 Seed-associated fungal assemblages are impacted by $\boldsymbol{A} \boldsymbol{b}$ Abra43 transmission

282 The structure of 148 microbial assemblages associated to seeds harvested in C2013, C2014, 283 A2013, A2014, X2013 and X2014 was assessed through amplification and subsequent sequencing 284 of two bacterial molecular markers (16S rRNA gene and $g y r B)$ and one fungal molecular marker 285 (ITS1). A total of 7,870,622 (16S rRNA gene), 24,355,191 (gyrB) and 8,799,598 (ITS1) paired286 end reads were obtained (Table S2). Reads were assembled in quality sequences and grouped into 287 operational taxonomic units (OTUs) at $\geq 97 \%$ sequence identity for $16 \mathrm{~S}$ rRNA gene and ITS1 288 sequences and $\geq 98 \%$ sequence identity for $g y r B$. (Table S2). To increase the reproductibility of OTU detection between samples, OTUs with a relative abundance $\geq 0.1 \%$ of the library size were 290 defined as abundant OTUs (Barret et al., 2015). However, this threshold remove rare OTUs, which contributes to a large amount of diversity observed within microbial assemblages (Shade et al., 2014). Therefore, subsequent analyses were performed $(i)$ on every OTUs including abundant and rare OTUs and (ii) on abundant OTUs (aOTUs) only. Since seed samples used in this study have been either harvested manually on individual plants or mechanically with a threshing machine (see experimental procedures for further informations), the influence of the harvesting method on the structure of seed-associated microbial assemblages was first investigated. According to ANOSIM tests, seed samples harvested manually are not significantly different from samples collected with 
298 a threshing machine, suggesting that the harvesting method did not impact the structure of

299 microbial assemblages (Table 1).

300 The impact of pathogen transmission was evaluated on microbial richness using OTU and

301 aOTU counts as proxies for species richness. Overall, the number of bacterial OTU (Fig. S2A and

302 Fig. S2B) and aOTU (Fig. 2A and Fig. 2B) was constant between control samples and seed

303 harvested from A2013, A2014, X2013 and X2014. Therefore, the presence of Xcc 8004 and $A b$

304 Abra43 within the seed microbiota does not seem to alter bacterial richness. While fungal richness

305 was also not affected by seed transmission of Xcc 8004, the number of fungal OTU and aOTU

306 significantly $(P<0.01$, as assessed by ANOVA with post hoc Tukey's HSD test) decreased for

307 A2013 and A2014 (Fig. 2C and Fig. S2C). Hence $A b$ Abra43 seems to reduce the number of

308 resident fungal taxa associated to radish seeds.

309 We next measured the effect of $X c c$ and $A b$ transmission on microbial diversity. According

310 to inverse Simpson diversity index, bacterial $\alpha$-diversity was neither affected by $X c c 8004$ nor $A b$

311 Abra43 transmission (Fig. 2D, Fig. 2E, Fig. S2D and Fig. S2E). Changes in resident bacterial

312 assemblages between samples was further estimated by Bray-Curtis dissimilarity measure (Bray

313 and Curtis, 1957) and unweighted UniFrac distance (Lozupone and Knight, 2005) using OTU and

314 aOTU counts obtained with 16S rRNA gene and gyrB sequences. According to hierarchical

315 clustering and ANOSIM tests, seeds harvested from inoculated plants were not significantly

316 different from seeds collected in the control plot (Fig. S3, Fig. S4 and Table 1). This suggests that

317 neither $X c c 8004$ nor $A b$ Abra43impacted the structure of seed-associated bacterial assemblages.

318 Regarding seed-associated fungal assemblages, no significant difference in $\alpha$-diversity was

319 observed between control seed samples and samples contaminated with Xcc (Fig. 2F and Fig.

320 S2F). However, a significant reduction of inverse Simpson diversity index was observed following 
321 invasion of seed-associated fungal assemblages by $A b$ (Fig. 2F and Fig. S2F). To gain more insight

322 on the influence of $A b$ on seed-associated fungal assemblages, $\beta$-diversity was estimated with

323 Bray-Curtis dissimilarity measure. A significant disturbance $(p<0.001)$ of seed-associated fungal

324 assemblages was observed for samples harvested in A2013 and A2014 (Fig. 3 and Table 1).

325 Indeed all these seed samples grouped together, which indicates that transmission of $A b$ Abra43

326 from plant to seed has a profound influence on the structure of seed-associated fungal assemblages

327 (Fig. 3). According to canonical analysis of principal coordinates (CAP), the seed transmission of

$328 A b$ was explaining $63 \%(p<0.001)$ of the variation in fungal diversity across seed samples.

\section{Shift in relative abundance of microbial taxa following seed transmission of $A b$ Abra43}

331 The taxonomic composition of seed-associated microbial assemblages was investigated in samples

332 harvested in C2013 and C2014. According to both 16S rRNA gene and gyrB sequences, bacterial

333 aOTUs were mostly affiliated to Enterobacteriales and Pseudomonadales (Fig. S5), which

334 confirms that taxonomic classification performed with these two molecular markers give similar

335 results at high taxonomic rank (e.g. order level). Distribution of bacterial aOTU was then

336 investigated across seed samples. Only 3 16S rRNA aOTUs affiliated to Pantoea (Otu00001) and

337 Pseudomonas (Otu00002 and Otu00003) were shared across all seed samples (Fig. S3). Three

338 gyrB aOTUs corresponding to Pantoea agglomerans (Otu00001), Pseudomonas viridiflava

339 (Otu00002) and Erwinia tasmaniensis (Otu00003) were also conserved between all samples (Fig.

340 S4). These aOTUs were highly-abundant in all seed samples with an average relative abundance

341 of 58\% (P. agglomerans), 12\% (P. viridiflava) and 4\% (E. tasmaniensis) of all gyrB sequences.

342 While we did not identify bacterial aOTUs specifically associated to C, A and X samples,

343 significant changes ( $p$-value $\leq 0.05$ and LDA score $\geq 2$ ) in relative abundance of bacterial aOTUs 
344 were observed with LEfSE (Segata et al., 2011). Unsurprisingly, the relative abundance of aOTUs

345 affiliated to Xanthomonas (Otu0004 - 16S rRNA gene sequences) and Xanthomonas campestris

346 (Otu00039 - gyrB sequences) were both increased in seed samples harvested from X2013 and

347 X2014 (Table S3 and Table S4). The increase in relative abundance of these Xanthomonas-related

348 aOTUs was associated with changes in relative abundance of bacterial aOTUs belonging to the

349 Pseudomonodaceae and Enterobacteriaceae (Table S3 and Table S4) and of fungal aOTUs mainly 350 related to Alternaria (Table S5).

Regarding fungal assemblage composition, the seed microbiota of C2013 and C2014

352

353

354

355

356

357

358

359

360

361

362

363

364

365

366 samples was mainly composed of Pleosporales (Fig. S5). A significant shift $(p<0.01)$ in relative abundance of this fungal order was observed in A2013 and A2014 samples (Fig. S5). Since $A b$ belonged to the Pleosporales, this increase is probably due to the seed transmission of this pathogenic agent. At the aOTU-level 8 fungal entities were conserved in all seed samples (Fig. 3), five of them were affiliated to Pleosporales, two to Capnodiales and one to Basidiomycota. According to LEfSE analysis, 7 aOTUs were enriched in A2013 and 2014 samples (Fig. 3 and Table S5). These entities belonged to Alternaria and three of them are affiliated to Alternaria sect. brassicicola (OTU0443, OTU0446 and OTU0937). However, closer examination of the representative sequences of these aOTUs revealed that Abra43 belonged to OTU0446 and is the dominant phylotype of this group (96\% of all reads included in OTU0446). Seed transmission of Abra43 also results in decrease in relative abundance of 61 fungal aOTUs that belonged to various fungal genus including Alternaria, Cladosporium or Fusarium (Table S5). In addition 7 (16S rRNA gene sequences) and 14 (gyrB sequences) bacterial aOTUs were also impacted by Abra43 transmission (Table S3 and Table S4). 


\section{Correlation between microbial taxa within the seed microbiota}

368 In order to predict microbial interactions within seed-associated assemblages, we explored positive

369

370

371

372

373

374

375

376

377

378

380

381

382

383

384

385

386

387

388

389 and negative associations between entities of these assemblages by generating correlations networks with SparCC (Friedman and Alm, 2012). Considering only inferred correlations with pseudo $p$-values $\leq 0.001$, we identified 15 and 100 nodes in control samples with $16 \mathrm{~S}$ and $g y r B$ aOTUs, respectively (Fig. 4, Fig. S6 and Table S6). These nodes were sharing a total of 52 edges with $16 \mathrm{~S}$ aOTUs and 206 edges with $g y r B$ aOTUs. The other bacterial correlations networks generated with samples from $\mathrm{A}$ and $\mathrm{X}$ plots were not different from the network inferred with samples from control plots (Fig. 4, Fig. S6 and Table S6). Indeed all bacterial networks were split into multiples small modules with low connectivity between nodes. Moreover these inferred correlation networks were characterized by nodes having a maximal degree of 4 to 7 edges for 16S OTUs and 4 to 6 connections for gyrB aOTUs.

In comparison to bacterial networks, correlations networks inferred from seed-associated fungal assemblages were composed of more edges and less modules (Fig. 4 and Table S5), suggesting more interactions between entities of these assemblages. Seed transmission of Xcc 8004 did not impact the overall structure of the inferred fungal network, since the number of nodes, edges and median number of connectivity were constant between samples from $\mathrm{C}$ and $\mathrm{X}$ plots. Moreover, the highest degree nodes of both networks were related to fungal aOTUs conserved in each seed samples such as OTU0449 (Alternaria brassicae) for C and OTU0536 (Alternaria sect. infectoria) for X plots. On the contrary, Ab Abra43 strongly impacted the topology of the network with a decrease of nodes, edges and median number of connectivity (Fig. 4 and Table S6). Although OTU0449 and OTU0536 are still part of the fungal network in samples from A plots, these entities are not hubs anymore and shared 2 and 5 connections with other nodes, respectively. 
391 between the bacterial aOTU affiliated to $X c c$ and other entities of seed-associated bacterial 392 assemblages. Analysis of inferred correlation between $A b$ (OTU0446) and other fungal aOTUs 393 revealed positive correlations with 7 entities that belong exclusively to Alternaria. Four of these 394 aOTUs were already highlighted by hierarchical clustering (Fig. 3). In addition, 12 negative 395 correlations between OTU0446 and other fungal entities were observed. Some of these fungal 396 aOTUs were affiliated to Alternaria but also to other fungal orders such as Filobasidiales and 397 Sporidiobolales. Interestingly the highest degree node of the correlation network inferred in 398 samples harvested from A plot (OTU0309) was negatively associated to $A b$, suggesting that seed 399 transmission of this phytopathogenic fungus severely impacted resident fungal community. 


\section{Discussion (833 words)}

402 The aim of the present study was to assess the influence of two microbial invaders, namely

403

404

405

406

407

408

409

410

411

412

413

414

415

416

417

418

419

420

421

422

423

Xanthomonas campestris pv. campestris (Xcc) 8004 and A. brassicicola (Ab) Abra43, on the structure of resident microbial assemblages associated with seeds. According to our indicators of bacterial (16S rRNA gene and gyrB sequences) and fungal (ITS1) diversity, seed transmission of the bacterial strain Xcc 8004 did not impact the overall composition of seed-associated microbial assemblage. In contrast, transmission of Abra43 significantly changed the structure of resident fungal assemblages without altering bacterial assemblages' composition.

Variation in response of resident microbial assemblages to invasion of Xcc 8004 and Abra43 could be explained by the distinct transmission pathways employed by these two microorganisms to invade seeds, which ultimately result in differences of spatio-temporal distribution of these strains within the seed habitat. $X c c$ has been reported to invade seeds via the vascular tissue of the mother plant (Cook et al., 1952) or by flower infection during pollination (Van Der Wolf and Van Der Zouwen, 2010). Therefore, successful seed transmission of Xcc is probably strongly dependent of local resources available in the plant xylem or on flower surface. Conversely, $A b$ invades the seed via colonization of the pods and subsequent migration through the funicles (Singh and Mathure, 2004) and thus interacted mostly with microbial taxa associated to pods. As a consequence of these different routes of infection, Xcc is mostly located in the endosperm or inner integuments (Maude, 1996), while $A b$ is frequently isolated from the hilum of the seed coat (Knox-Davies, 1979). Therefore the contrasted response of seed-associated microbial assemblages to invasion by $X c c$ or $A b$ could be due to differences in microbial interactions occurring within these distinct micro-habitats. Differences in seed transmission pathways between $X c c$ and $A b$ also results in distinct timing of seed colonization, $X c c$ being associated with earlier 
424 seed development stage. Recently, it has been hypothesized that assembly history may determine 425 the structure of seed-associated bacterial assemblages (Aleklett and Hart, 2013; Klaedtke et al., 426 2015) in a similar manner than other plant-related habitat such as the phyllosphere (Maignien et 427 al., 2014). Therefore, the resistance of seed-associated bacterial assemblage to Xcc 8004 and

428

429

430

431 Abra43 invasions could be due to prior colonization of the seed by pioneer bacterial entities, which maintain community structure (Shade et al., 2012). Although this hypothesis has to be tested experimentally through temporal survey of microbial assemblages during the different seed development stages, it is tempting to speculate that these pioneer species may be related to bacterial taxa such as Erwinia, Pseudomonas or Pantoea. Indeed these taxa are highly abundant within the seeds samples collected in this study and have been frequently observed in flower (Aleklett et al., 2014) and seeds (Johnston-Monje and Raizada, 2011; Links et al., 2014; Barret et al., 2015; Klaedtke et al., 2015) of several plant species.

Alternatively, we cannot rule out the possibility that the observed stability of microbial assemblage in response to $X c c 8004$ transmission is partly due to the low abundance of $X c c$ detected within seed samples contaminated with this bacterial strain. Although the incidence of transmission of $X c c$ on radish seeds is in accordance with previous studies (Cook et al., 1952; Van Der Wolf and Van Der Zouwen, 2010; van der Wolf et al., 2013) and reflected the natural seed transmission observed with other bacterial pathogens (Darrasse et al., 2007), the high prevalence of seeds not contaminated with Xcc in X2013 (94\%) and X2014 (72\%) samples together with a low $X c c$ population sizes may result in a high background signal that is likely to mask changes occurring within microbial assemblages. In comparison the high incidence and abundance of $A b$ within A2013 and A2014 seed samples allow detection of changes in microbial assemblages' structure. 

impacted the structure of fungal assemblages. Perturbation of fungal assemblage following $A b$ transmission is probably explained by competition between $A b$ and other functional equivalent species for resources and spaces (Burke et al., 2011). Indeed the transmission of $A b$ from plant to seed is correlated with a decrease in relative abundance of closely related entities that belong to Alternaria brassicae, Alternaria. sect. infectoria and Alternaria. sect. alternate (Woudenberg et 453 al., 2013). As these fungal aOTUs represented hubs of inferred fungal correlation network in native condition, the potential competition of these entities with $A b$ result in a drastic shift in the structure of seed-associated fungal assemblage. Among the observed changes, we observed multiple cooccurrences between $A b$ and other fungal entities related to Alternaria sect. brassicicola. Whether these entities either interact positively or are selected in similar ways by the environment remained 458 to be determined.

The results of this work provide a first glimpse into the response of the seed microbiota 460 following seed transmission of two phytopathogenic microorganisms. Future metagenomics analysis of seed-associated microbial assemblages will be useful to assess the relationship between assemblage structure and function (Vayssier-Taussat et al., 2014). This research might lead to the development of biocontrol strategies based on the potential of seed-associated microbial 464 community. 


\section{Acknowledgments}

467 The authors wish to thanks Julie Gombert and Vincent Odeau (FNAMS) for their help with all the 468 field experiments, Muriel Bahut and Laurence Hibrand-Saint Oyant from the platform ANAN of 469 SFR Quasav for their help on the MiSeq experiments.

470 


\section{References}

Abarenkov, K., Henrik Nilsson, R., Larsson, K.H., Alexander, I.J., Eberhardt, U., Erland, S., Hoiland, K., Kjoller, R., Larsson, E., Pennanen, T., Sen, R., Taylor, A.F.S., Tedersoo, L., Ursing, B.M., Vralstad, T., Liimatainen, K., Peintner, U., and Koljalg U. (2010) The UNITE database for molecular identification of fungi--recent updates and future perspectives. New Phytol 186: 281-285.

Aleklett, K., and Hart, M. (2013) The root microbiota - a fingerprint in the soil? Plant and Soil 370: 671686.

Aleklett, K., Hart, M., and Shade, A. (2014) The microbial ecology of flowers: an emerging frontier in phyllosphere research. Botany 92: 253-266.

Avenot, H., Dongo, A., Bataillé-Simoneau, N., Iacomi- Vasilescu, B., Hamon, B., Peltier, D., and Simoneau, P. (2005) Isolation of 12 polymorphic microsatellite loci in the phytopathogenic fungus Alternaria brassicicola. Mol Ecol Notes 5: 948-950.

Bacilio-Jimenez, M., Aguilar-Flores, S., del Valle, M.V., Perez, A., Zepeda, A., and Zenteno, E. (2001) Endophytic bacteria in rice seeds inhibit early colonization of roots by Azospirillum brasilense. Soil Biol Biochem 33: 167-172.

Baker, K.F., and Smith, S.H. (1966) Dynamics of Seed Transmission of Plant Pathogens. Ann Rev Phytopathol4: 311-332.

Barret, M., Briand, M., Bonneau, S., Préveaux, A., Valière, S., Bouchez, O., Hunault, G., Simoneau, P., and Jacques, M.-A. (2015) Emergence Shapes the Structure of the Seed Microbiota. Appl Environ Microbiol 81: 1257-1266.

Bengtsson-Palme, J., Ryberg, M., Hartmann, M., Branco, S., Wang, Z., Godhe, A., De Wit, P., SanchezGarcia, M., Ebersberger, I., de Sousa, F., Amend, A., Jumpponen, A., Unterseher, M., Kristiansson, E., Abarenkov, K.; Bertrand Y., Sanli, K., Eriksson, M., Vik, U., Veldre, V., and Nilsson, H. (2013) Improved software detection and extraction of ITS1 and ITS2 from ribosomal ITS sequences of fungi and other eukaryotes for analysis of environmental sequencing data. Meth Ecol Evol 4: 914-919.

Bray, J.R., and Curtis, J.T. (1957) An Ordination of the Upland Forest Communities of Southern Wisconsin. Ecol Monograph 27: 325-349.

Burke, C., Steinberg, P., Rusch, D., Kjelleberg, S., and Thomas, T. (2011) Bacterial community assembly based on functional genes rather than species. Proc Nat Acad Sci 108: 14288-14293.

Carbone, I., and Kohn, L. (1999) A method for designing primer sets for speciation studies in filamentous ascomycetes. Mycologia 91: 553-556.

Cole, J.R., Wang, Q., Cardenas, E., Fish, J., Chai, B., Farris, R.J., Kulam-Syed-Mohideen, A.S., McGarrell, D.M., Marsh, T., Garrity, G.M., and Tiedje, J.M.. (2009) The Ribosomal Database Project: improved alignments and new tools for rRNA analysis. Nucleic Acids Res 37: D141-145.

Cook, A.A., Larson, R.H., and Walker, J.C. (1952) Relation of the black rot pathogen to cabbage seed. Phytopathol 42: 316-320.

Darrasse, A., Bureau, C., Samson, R., Morris, C., and Jacques, M.-A. (2007) Contamination of bean seeds by Xanthomonas axonopodis pv. phaseoli associated with low bacterial densities in the phyllosphere under field and greenhouse conditions. Eur J Plant Pathol 119: 203-215.

Darrasse, A., Darsonval, A., Boureau, T., Brisset, M.-N., Durand, K., and Jacques, M.-A. (2010) Transmission of Plant-Pathogenic Bacteria by Nonhost Seeds without Induction of an Associated Defense Reaction at Emergence. Appl Environ Microbiol 76: 6787-6796.

Darsonval, A., Darrasse, A., Durand, K., Bureau, C., Cesbron, S., and Jacques, M.A. (2009) Adhesion and fitness in the bean phyllosphere and transmission to seed of Xanthomonas fuscans subsp. fuscans. Mol Plant Microbe Interact 22: 747-757.

Darsonval, A., Darrasse, A., Meyer, D., Demarty, M., Durand, K., Bureau, C., Manceau, C., and Jacques, M.-A. (2008) The Type III secretion system of Xanthomonas fuscans subsp. fuscans is involved in the phyllosphere colonization process and in transmission to seeds of susceptible beans. Appl Environ Microbiol 74: 2669-2678. 
Dekkers, B.W., Costa, M., Maia, J., Bentsink, L., Ligterink, W., and Hilhorst, H.M. (2015) Acquisition and loss of desiccation tolerance in seeds: from experimental model to biological relevance. Planta 241: 563577.

Dutta, B., Avci, U., Hahn, M.G., and Walcott, R.R. (2012) Location of Acidovorax citrulli in infested watermelon seeds is influenced by the pathway of bacterial invasion. Phytopathol 102: 461-468.

Dutta, B., Gitaitis, R., Smith, S., and Langston, D., Jr. (2014) Interactions of seedborne bacterial pathogens with host and non-host plants in relation to seed infestation and seedling transmission. PLoS One 9: e99215. Edgar, R.C. (2010) Search and clustering orders of magnitude faster than BLAST. Bioinformatics 26: 24602461.

Edgar, R.C., Haas, B.J., Clemente, J.C., Quince, C., and Knight, R. (2011) UCHIME improves sensitivity and speed of chimera detection. Bioinformatics 27: 2194-2200.

Epskamp, S., Cramer, A.O.J., Waldorp, L.J., Schmittmann, V.D., and Borsboom, D. (2012) qgraph: Network Visualizations of Relationships in Psychometric Data. J Stat Sof 48: 1-18.

Friedman, J., and Alm, E.J. (2012) Inferring Correlation Networks from Genomic Survey Data. PLoS Comput Biol 8: e1002687.

Gitaitis, R., and Walcott, R. (2007) The epidemiology and management of seedborne bacterial disease. Annu Rev Phytopathol 45:371-397.

Goggin, D.E., Emery, R.J., Kurepin, L.V., and Powles, S.B. (2015) A potential role for endogenous microflora in dormancy release, cytokinin metabolism and the response to fluridone in Lolium rigidum seeds. Ann Bot 115: 293-301.

Hacquard, S., Garrido-Oter, R., González, A., Spaepen, S., Ackermann, G., Lebeis, S., McHardy, A.C., Dangl, J.L., Knight, R., Ley, R., and Schulze-Lefert, P. (2015) Microbiota and Host Nutrition across Plant and Animal Kingdoms. Cell Host \& Microbe 17: 603-616.

Iacomi-Vasilescu, B., Bataille-Simoneau, N., Campion, C., Dongo, A., Laurent, E., Serandat, I., Hamon, B., and Simoneau, P. (2008) Effect of null mutations in the AbNIK1 gene on saprophytic and parasitic fitness of Alternaria brassicicola isolates highly resistant to dicarboximide fungicides. Plant Pathol 57: 937-947.

Johnston-Monje, D., and Raizada, M.N. (2011) Conservation and Diversity of Seed Associated Endophytes in Zea across Boundaries of Evolution, Ethnography and Ecology. PLoS ONE 6: e20396.

Jones, J.D., and Dangl, J.L. (2006) The plant immune system. Nature 444: 323-329.

Jousset, A., Schulz, W., Scheu, S., and Eisenhauer, N. (2011) Intraspecific genotypic richness and relatedness predict the invasibility of microbial communities. ISME J 5: 1108-1114.

Klaedtke, S., Jacques, M.-A., Raggi, L., Préveaux, A., Bonneau, S., Negri, V., Chable, V., and Barret, M. (2015) Terroir is a key driver of seed-associated microbial assemblages. Environ Microbiol: doi: 10.1111/1462-2920.12977.

Knox-Davies (1979) Relationships between Alternaria brassicicola and Brassica seeds. Trans Brit Mycol Soc 73: 235-248.

Kozich, J.J., Westcott, S.L., Baxter, N.T., Highlander, S.K., and Schloss, P.D. (2013) Development of a dual-index sequencing strategy and curation pipeline for analyzing amplicon sequence data on the MiSeq Illumina sequencing platform. Appl Environ Microbiol 79: 5112-5120.

Links, M.G., Demeke, T., Grafenhan, T., Hill, J.E., Hemmingsen, S.M., and Dumonceaux, T.J. (2014) Simultaneous profiling of seed-associated bacteria and fungi reveals antagonistic interactions between microorganisms within a shared epiphytic microbiome on Triticum and Brassica seeds. New Phytol 202: 542-553.

Lopez-Velasco, G., Carder, P.A., Welbaum, G.E., and Ponder, M.A. (2013) Diversity of the spinach (Spinacia oleracea) spermosphere and phyllosphere bacterial communities. Fems Microbiol Lett 346: 146154.

Lozupone, C., and Knight, R. (2005) UniFrac: a new phylogenetic method for comparing microbial communities. Appl Environ Microbiol 71: 8228-8235. 
Maignien, L., DeForce, E.A., Chafee, M.E., Eren, A.M., and Simmons, S.L. (2014) Ecological Succession and Stochastic Variation in the Assembly of Arabidopsis thaliana Phyllosphere Communities. mBio 5: e00682-13.

Mallon, C.A., Elsas, J.D., and Salles, J.F. (2015a) Microbial Invasions: The Process, Patterns, and Mechanisms. Trends Microbiol 23: 719-729.

Mallon, C.A., Poly, F., Le Roux, X., Marring, I., van Elsas, J.D., and Salles, J.F. (2015b) Resource pulses can alleviate the biodiversity-invasion relationship in soil microbial communities. Ecology 96: 915-926. Markowitz, V.M., Chen, I.-M.A., Palaniappan, K., Chu, K., Szeto, E., Grechkin, Y., Ratner, A., Jacob, B., Pati, A., Huntemann, M., Liolios, K., Pagani, I., Anderson, I., Mavromatis, K., Ivanova, N.N., and Kyrpides, C. (2012) IMG: the integrated microbial genomes database and comparative analysis system. Nucl Acids Res 40: D115-D122.

Maude, R.B. (1996) Seedborne diseases and their control: principles and practice.

McMurdie, P.J., and Holmes, S. (2014) Waste Not, Want Not: Why Rarefying Microbiome Data Is Inadmissible. PLoS Comput Biol 10: e1003531.

Mendes, R., Kruijt, M., de Bruijn, I., Dekkers, E., van der Voort, M., Schneider, J.H., Piceno, Y.M., DeSantis, T.Z., Andersen, G.L., Bakker, P.A.H.M, and Raiijmakers, J.M. (2011) Deciphering the rhizosphere microbiome for disease-suppressive bacteria. Science 332: 1097-1100.

Nelson, E.B. (2004) Microbial dynamics and interactions in the spermosphere. Ann Rev Phytopathol 42: 271-309.

Ngugi, H.K., and Scherm, H. (2006) Biology of flower-infecting fungi. Ann Rev Phytopathol 44: 261-282. Pochon, S., Terrasson, E., Guillemette, T., Iacomi-Vasilescu, B., Georgeault, S., Juchaux, M., Berruyer, R., Debeaujon, I., Simoneau, P., and Campion, C. (2012) The Arabidopsis thaliana-Alternaria brassicicola pathosystem: a model interaction for investigating seed transmission of necrotrophic fungi. Plant Meth 8: 16.

Pochon, S., Simoneau, P., Pigne, S., Balidas, S., Bataille-Simoneau, N., Campion, C., Jaspard, E., Calmes, B., Hamon, B., Berruyer,R., Juchaux, M., and Guillemette, T. (2013) Dehydrin-like proteins in the necrotrophic fungus Alternaria brassicicola have a role in plant pathogenesis and stress response. PLoS One 8: e75143.

Qian, W., Jia, Y., Ren, S.X., He, Y.Q., Feng, J.X., Lu, L.F., Sun, Q., Ying, G., Tang, D.J., Tang, H., Wu, W., Hao, P., Wang, L., Jiang, B.L., Zeng, S., Gu, W.Y., Lu, G., Rong, L., Tian, Y., Yao, Z., Fu, G., Chen, B., Fang, R., Qiang, B., Chen, Z., Zhao, G.P., Tang, J.L., and He, C. (2005) Comparative and functional genomic analyses of the pathogenicity of phytopathogen Xanthomonas campestris pv. campestris. Genome Res 15: 757-767.

Rijlaarsdam, A., Woudt, B., Simons, G., Koenraadt, H., Oosterhof, J., Asma, M., Buddiger, P., Roorda, P., Grimault, V., and de Koning, J. (2004) Development of specific primers for the molecular detection of Xanthomonas campestris pv. campestris. In EPPO Conference on Quality of Diagnosis and New Diagnostic Methods for Plant Pests. Noordwijkerhout, the Netherlands.

Schloss, P.D., Westcott, S.L., Ryabin, T., Hall, J.R., Hartmann, M., Hollister, E.B., Lesniewski, R.A., Oakley, B.B., Parks, D.H;, Robinson, C.J., Sahl, J.W., Stres, B., Thallinger, G.G., Van Horn, D.J., and Weber, C.F. (2009) Introducing mothur: Open-Source, Platform-Independent, Community-Supported Software for Describing and Comparing Microbial Communities. Appl Environ Microbiol 75: 7537-7541. Segata, N., Izard, J., Waldron, L., Gevers, D., Miropolsky, L., Garrett, W.S., and Huttenhower, C. (2011) Metagenomic biomarker discovery and explanation. Genome Biol 12: R60.

Shade, A., McManus, P.S., and Handelsman, J. (2013) Unexpected diversity during community succession in the apple flower microbiome. MBio 4: e00602-12.

Shade, A., Jones, S.E., Caporaso, J.G., Handelsman, J., Knight, R., Fierer, N., and Gilbert, J.A. (2014) Conditionally rare taxa disproportionately contribute to temporal changes in microbial diversity. MBio 5: e01371-01314.

Shade, A., Peter, H., Allison, S.D., Baho, D.L., Berga, M., Burgmann, H., Huber, D.H., Langenheder, S., Lennon, J;T., Martiny, J.B., Matulich, K.L., Schmidt, T.M., and Handelsman, J. (2012) Fundamentals of microbial community resistance and resilience. Front Microbiol 3: 417. 
620 Singh, D., and Mathure, S. (2004) Location of Fungal Hyphae in Seeds. In Histopathology of Seed-Borne 621 Infections: CRC Press, pp. 101-168.

622 Spinelli, F., Ciampolini, F., Cresti, M., Geider, K., and Costa, G. (2005) Influence of stigmatic morphology on flower colonization by Erwinia amylovora and Pantoea agglomerans. Eur J Plant Pathol 113: 395-405. Tancos, M.A., Chalupowicz, L., Barash, I., Manulis-Sasson, S., and Smart, C.D. (2013) Tomato Fruit and Seed Colonization by Clavibacter michiganensis subsp. michiganensis through External and Internal Routes. Appl Environ Microbiol 79: 6948-6957.

627 Terrasson, E., Darrasse, A., Righetti, K., Buitink, J., Lalanne, D., Ly Vu, B. Pelletier, S., Bolingue, W., 628 Jacques, M.A., and Leprince, O. (2015) Identification of a molecular dialogue between developing seeds of Medicago truncatula and seedborne xanthomonads. J Exp Bot doi: 10.1093/jxb/erv167.

630 Van Der Wolf, J.M., and Van Der Zouwen, P.S. (2010) Colonization of Cauliflower Blossom (Brassica 631 oleracea) by Xanthomonas campestris pv. campestris, via Flies (Calliphora vomitoria) Can Result in Seed 632 Infestation. Journal of Phytopathol 158: 726-732.

633 van der Wolf, J.M., van der Zouwen, P.S., and van der Heijden, L. (2013) Flower infection of Brassica 634 oleracea with Xanthomonas campestris pv. campestris results in high levels of seed infection. Eur J Plant 635 Pathol 136: 103-111.

636 van Elsas, J.D., Chiurazzi, M., Mallon, C.A., Elhottova, D., Kristufek, V., and Salles, J.F. (2012) Microbial 637 diversity determines the invasion of soil by a bacterial pathogen. Proc Natl Acad Sci U S A 109: 1159-1164. 638 van Overbeek, L.S., Franke, A.C., Nijhuis, E.H., Groeneveld, R.M., da Rocha, U.N., and Lotz, L.A. (2011) 639 Bacterial communities associated with Chenopodium album and Stellaria media seeds from arable soils. 640 Microb Ecol 62: 257-264.

641 Vayssier-Taussat, M., Albina, E., Citti, C., Cosson, J.-F., Jacques, M.-A., Lebrun, M.-H., Le Loir, Y., 642 Ogliastro, M., Petit, M.-A., Roumagnac, P., and Candresse, T. (2014). Shifting the paradigm from 643 pathogens to pathobiome : new concepts in the light of meta-omics. Front Cell Infect Microbiol 4:29.

644 Vorholt, J.A. (2012) Microbial life in the phyllosphere. Nat Rev Microbiol 10: 828-840.

645 Wang, Q., Garrity, G.M., Tiedje, J.M., and Cole, J.R. (2007) Naive Bayesian classifier for rapid assignment 646 of rRNA sequences into the new bacterial taxonomy. Appl Environ Microbiol 73: 5261-5267.

Wei, Z., Yang, T., Friman, V.P., Xu, Y., Shen, Q., and Jousset, A. (2015) Trophic network architecture of root-associated bacterial communities determines pathogen invasion and plant health. Nat Commun 6: 8413.

651

Woudenberg, J.H.C., Groenewald, J.Z., Binder, M., and Crous, P.W. (2013) Alternaria redefined. Stud Mycol 75: 171-212. 


\section{1}

Figure 1: Assessment of seed contamination by $X$. campestris pv. campestris 8004 and A. brassicicola Abra43

The contamination rate of seed samples by $X$. campestris pv. campestris 8004 (A) and $A$. brassicicola Abra43 (B) was assessed through microbiological analysis of 96 and 100 individual seeds, respectively. Contamination rates are the mean of 6 independent biological replicates performed for each experimental year. Quantitative detection of Xcc (C) was performed on seed samples harvested from uninoculated plants (C2013 and C2014) and plants inoculated with XcC (X2013 and X2014) through qPCR with primers targeting XC_1533.

Quantitative detection of $A b$ (D) was performed on seed samples harvested from uninoculated plants (C2013 and C2014) and plants inoculated with Ab (A2013 and A2014) through qPCR with primers and probe targeting $A b D h n 1$. The green lines represent the number of each target gene in the different samples, while bold dark black lines represent the median. The grey area indicates the density of distribution. Differences in contamination rate and relative abundance were considered significant at a $p$-value $\leq 0.01$ (as assessed by 2-sample test for equality of proportions and ANOVA with post hoc Tukey's HSD test, respectively). 
A
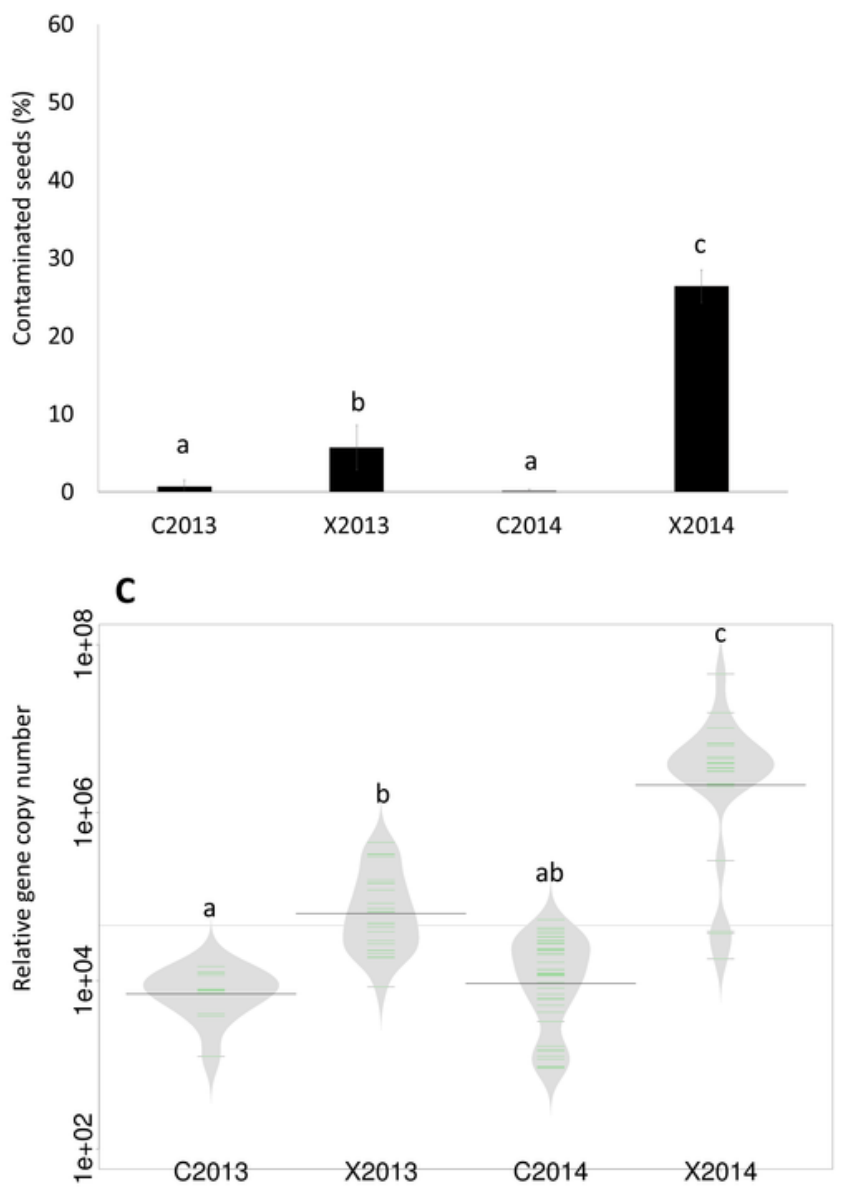
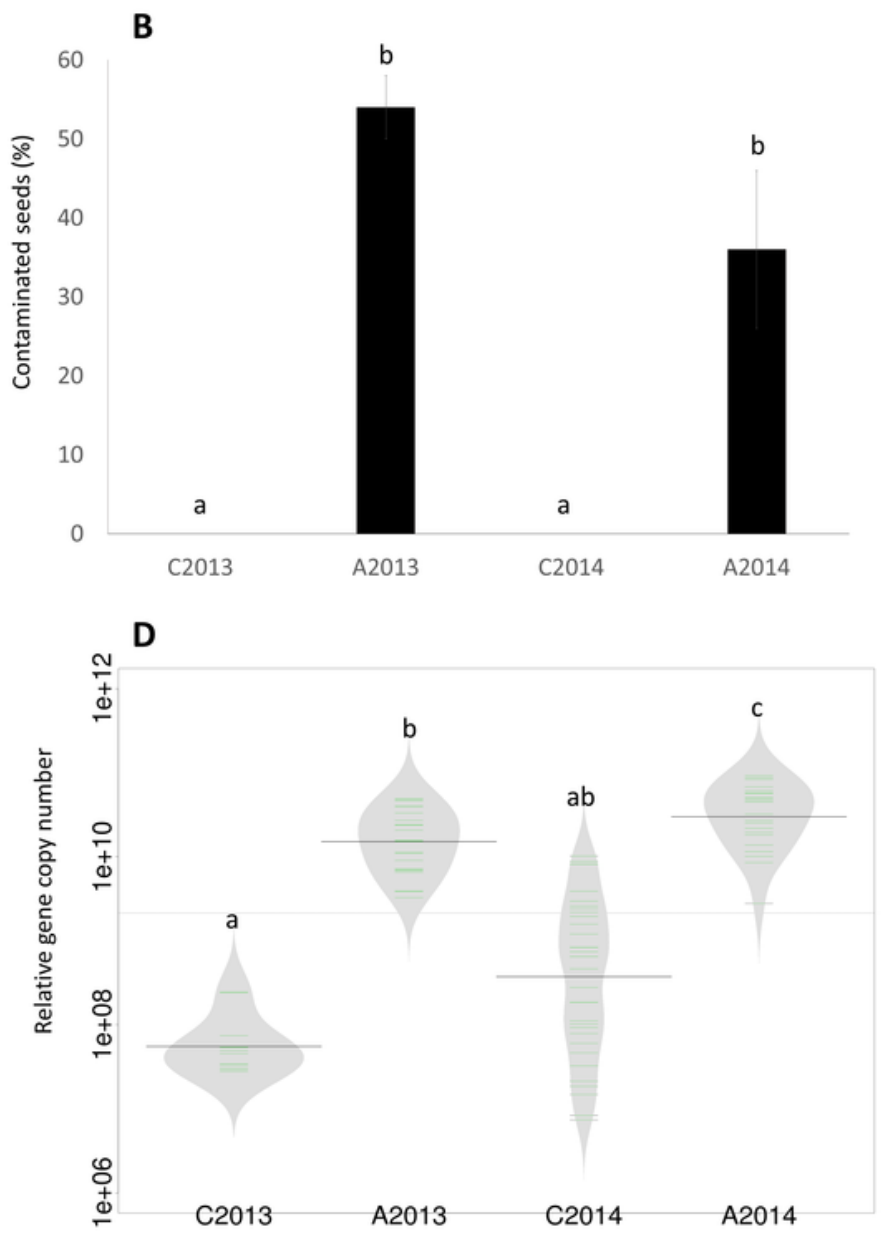
2

Figure 2: Richness and diversity of seed samples observed with abundant OTUs

Microbial richness ( $A, B$ and $C$ ) and diversity ( $D, E$ and $F$ ) were estimated with abundant OTUs obtained with 16S rRNA gene ( $A$ and $D)$, gyrB ( $B$ and $E$ ) and ITS1 sequences ( $C$ and F). Richness and diversity associated to uncontaminated seeds (C2013 and C2014), seeds contaminated with Xcc (X2013 and X2014) and seeds contaminated with Ab (A2013 and A2014) were compared. Each sample is represented by a green line, while black line represents the median. The grey area represents the density of distribution. Letters $a, b$ and c denote significant changes between conditions considered at a $P$-value $\leq 0.01$ (as assessed by ANOVA with post hoc Tukey's HSD test). 
A

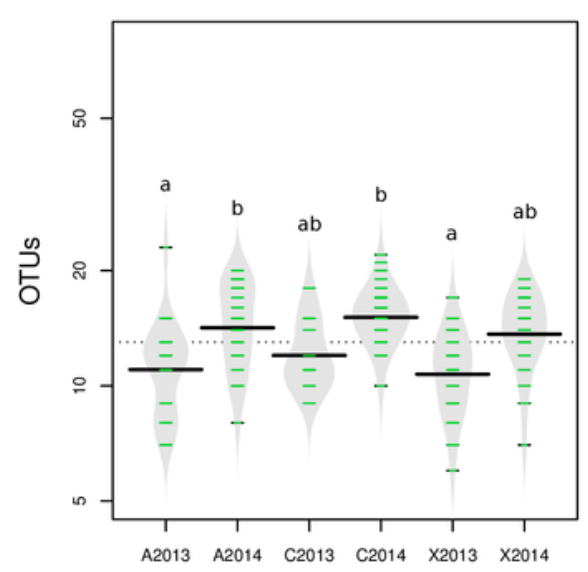

D

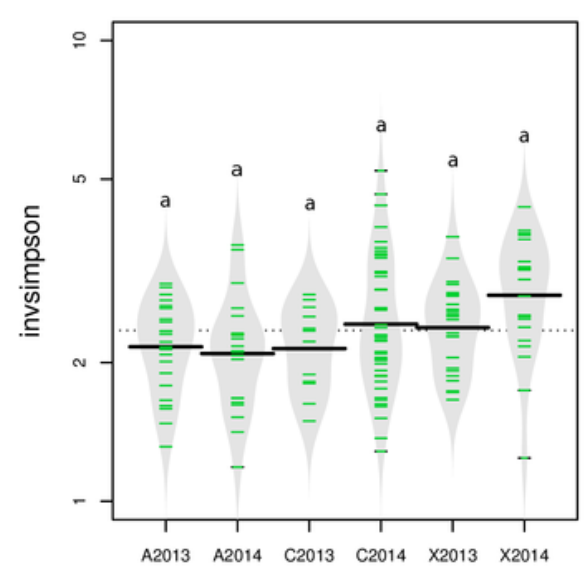

B

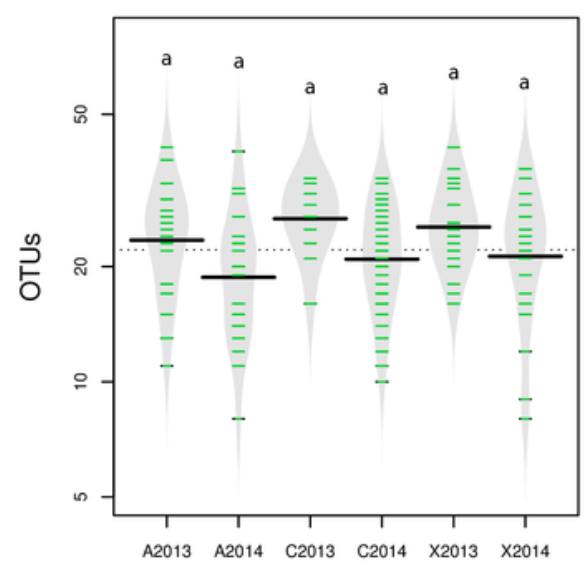

E

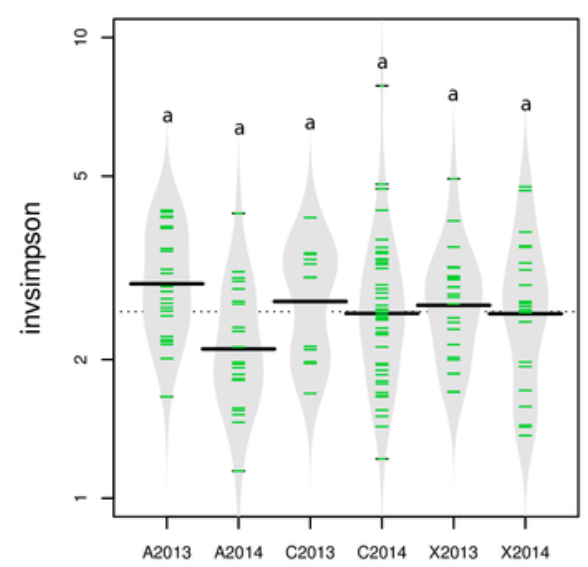

C

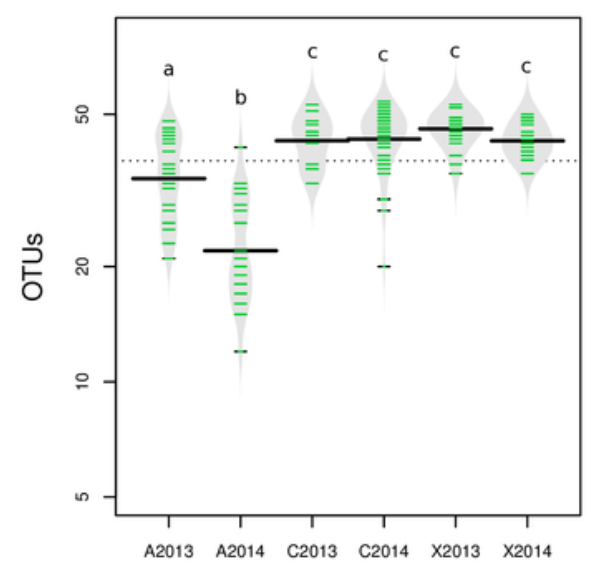

F

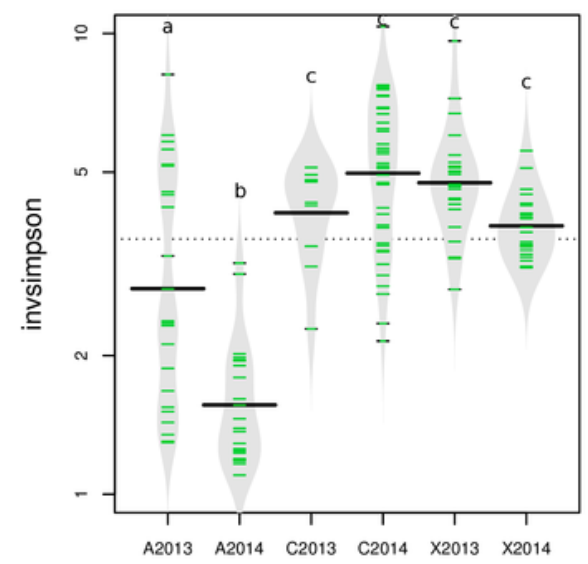




\section{3}

Figure 3: Influence of $A b$ on the structure of seed-associated fungal assemblages

Hierarchical clustering of seed samples ( $y$ axis) is based on Bray-Curtis dissimilarity measure. The type of samples is represented by gradual color changes: light blue for controls, medium blue for seeds contaminated with $X c c$ and dark blue for seeds contaminated with $A b$. Only abundant OTUs (threshold of $1 \%$ in relative abundance) are represented in the heatmap. These aOTUs are clustered by their co-occurrence patterns ( $x$ axis). According to analysis of similarity, a significant clustering of $A b$ seed samples was observed $(p<0.001)$.

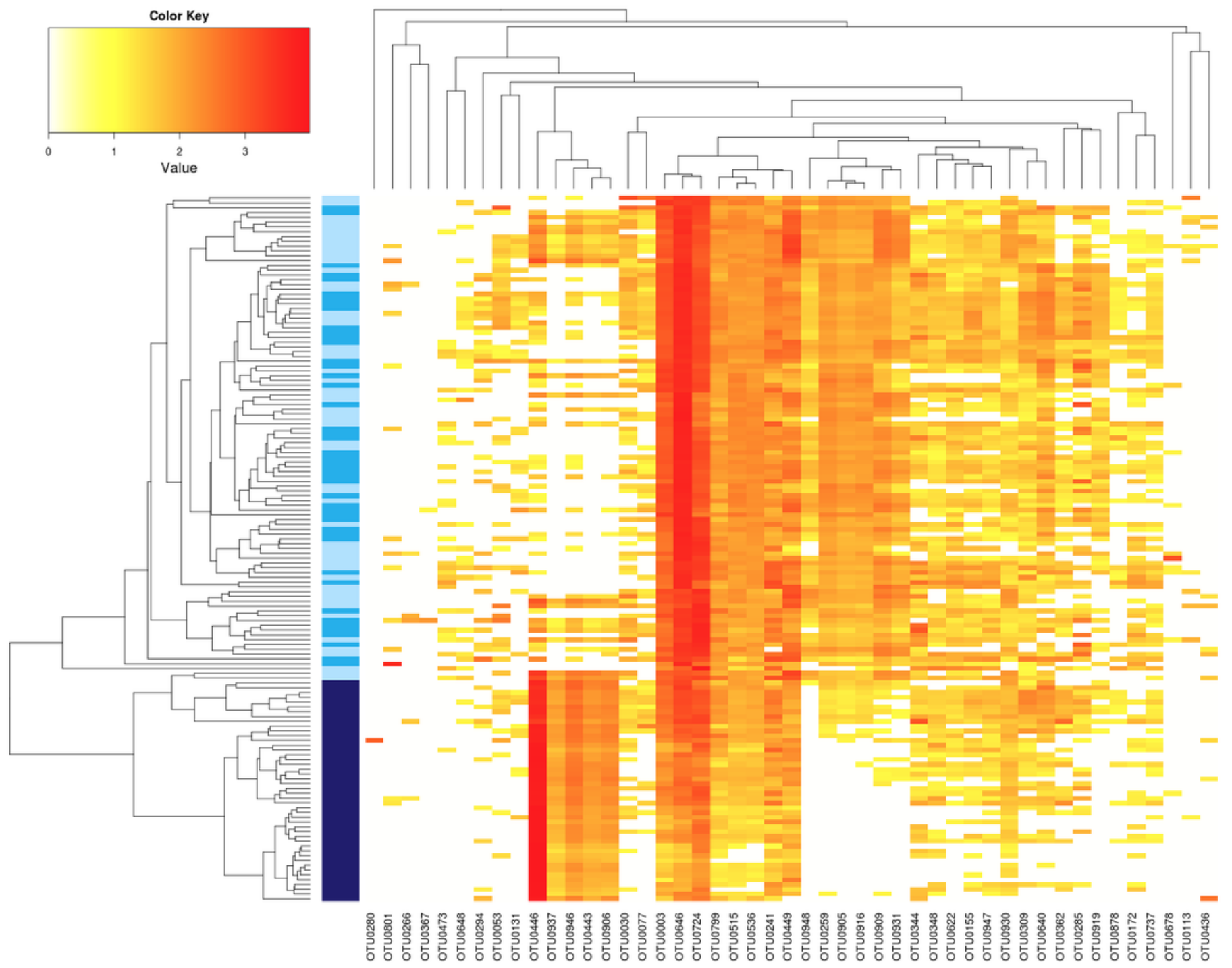




\section{4}

Figure 4: Inferred correlations between aOTUs

Correlation networks between bacterial taxa are based on gyrB sequences obtained in uncontaminated seeds (A), seeds contaminated with Xcc (B), and contaminated with $A b$ (C). Correlation networks between fungal taxa are based on ITS1 sequences obtained in uncontaminated seeds (D), seeds contaminated with $X c c(E)$, and contaminated with $A b(\mathrm{~F})$. Correlations between aOTUs were calculated with the Sparse Correlations for Compositional data algorithm. Each node represents an aOTUs, which is colored according to its taxonomic affiliation (family-level). Edges represent correlations between the nodes they connect with blue and orange colors indicating negative and positive inferred correlation, respectively. Only correlations with pseudo $p$-value $\leq 0.001$ were represented in the network using the $\mathrm{R}$ package qgraph. 
A

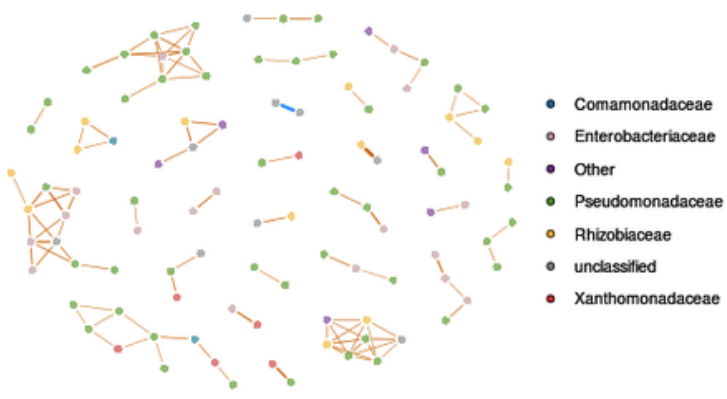

B

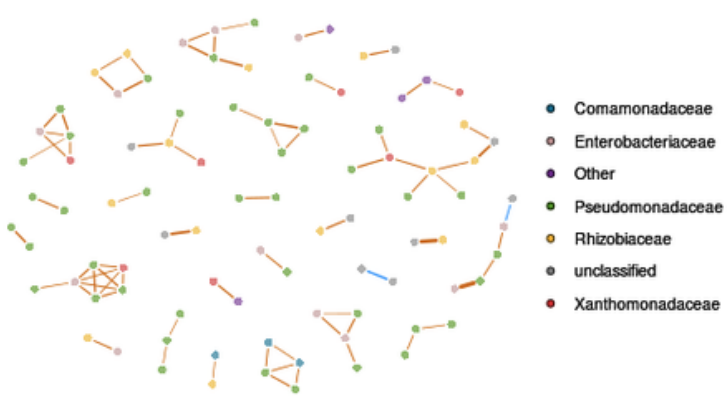

c

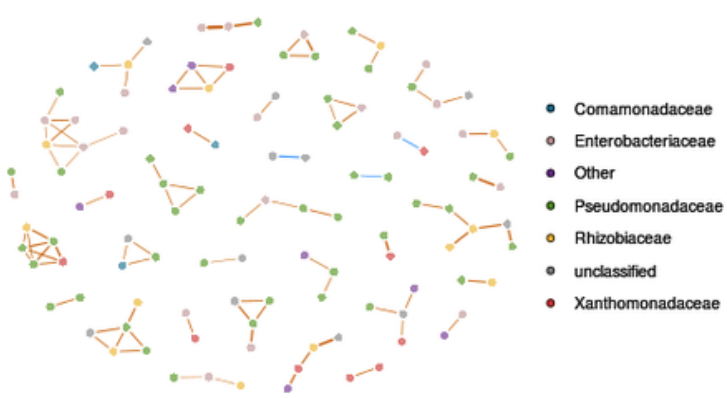

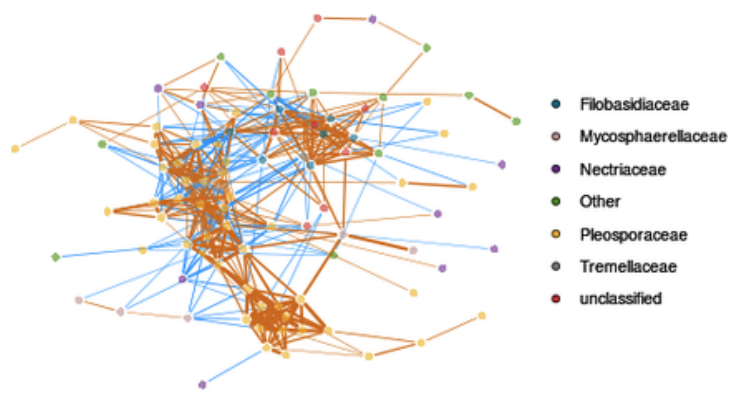

E

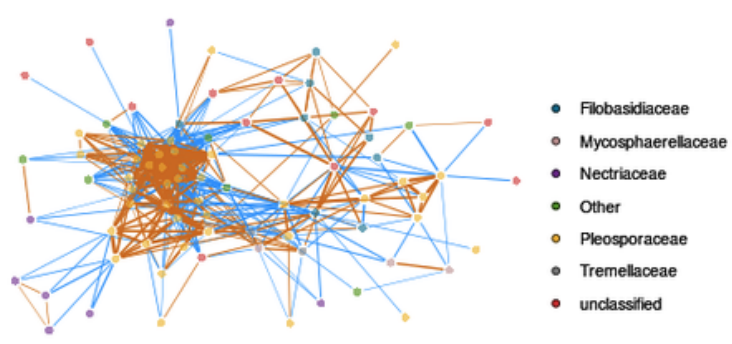

F

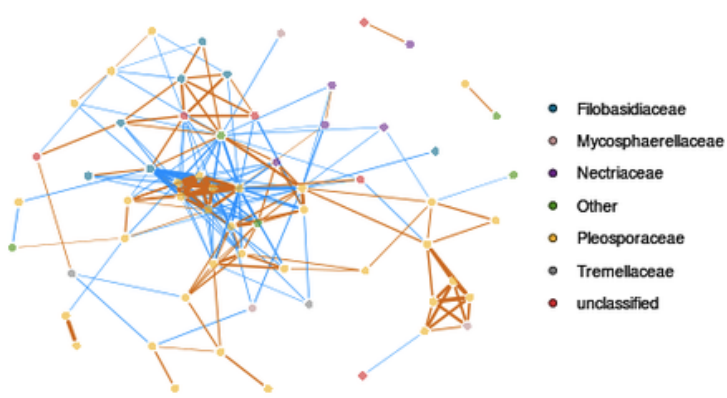




\section{Table $\mathbf{1}$ (on next page)}

Table 1: Analysis of similarity of seed-associated microbial assemblages

Analysis of similarity (ANOSIM) was used to assess the robustness of the hierarchical clustering analyses (Bray-Curtis dissimilarity measure and unweighted Unifrac distance). Pvalues are displayed in each column. Only $P$-values highlighted in bold are considered as significant 


\begin{tabular}{|c|c|c|c|c|c|c|c|}
\hline Marker & diversity index & OTU & $\mathrm{A} 13 \mathrm{vsC} 13$ & $\mathrm{X} 13 \mathrm{vsC} 13$ & $\mathrm{~A} 14 \mathrm{vsC} 14$ & $\mathrm{X} 14 \mathrm{vsC} 14$ & $\begin{array}{l}\text { Manual vs } \\
\text { Mechanical }\end{array}$ \\
\hline \multirow[t]{4}{*}{ 16S rRNA gene } & Bray-Curtis & OTUs & 0.693 & 0.249 & 0.932 & 0.045 & 0.991 \\
\hline & & aOTUs & 0.733 & 0.223 & 0.918 & 0.058 & 0.989 \\
\hline & Unifrac unweighted & OTUs & 0.698 & 0.298 & 0.032 & 0.029 & 0.976 \\
\hline & & aOTUs & 0.737 & 0.407 & 0.044 & 0.018 & 0.962 \\
\hline \multirow[t]{4}{*}{ gyrB } & Bray-Curtis & OTUs & 0.257 & 0.208 & 0.098 & 0.105 & 0.999 \\
\hline & & $\mathrm{aOTUs}$ & 0.156 & 0.138 & 0.041 & 0.028 & 0.998 \\
\hline & Unifrac unweighted & OTUs & 0.241 & 0.520 & 0.134 & 0.804 & 0.999 \\
\hline & & aOTUs & 0.203 & 0.467 & 0.026 & 0.111 & 0.957 \\
\hline \multirow[t]{2}{*}{ ITS1 } & Bray-Curtis & OTUs & $<0.001$ & 0.763 & $<0.001$ & 0.504 & 0.999 \\
\hline & & aOTUs & $<0.001$ & 0.777 & $<0.001$ & 0.406 & 0.999 \\
\hline
\end{tabular}

2 